\title{
Between ambiance and perception: Heritage decoding
}

\author{
Anetta Kepczynska-Walczak ${ }^{1, *}$ \\ ${ }^{1}$ Department of Digital Technologies in Architecture and Urban Planning, Institute of Architecture \\ and Urban Planning, Lodz University of Technology, Lodz (Łódź), Poland
}

\begin{abstract}
The aim of the study is to grasp and analyse the intangible process taking place between ambiance and perception. In this regard, ambiance should be understood as a state or aura, as opposed to perception, assumed as a process. The transition of sensorial immersion from ambiance to perception requires the process of decoding. Decoding might be explained as the process running between ambiance and perception and, furthermore, leading to understanding. Therefore, the outcome of the overall process is of cognitive characteristics. Furthermore, it draws research into a relationship between what is projected and actually experienced, between the tangible and the intangible and reveals the process of decoding ambiances where heritage plays a dominant role. The paper discusses the two concepts in the context of the post-industrial heritage in Lódź, Poland. The set of reflections evokes numerous questions: are we into the heritage as it was, or as it is at present, or rather to its projection, tuned to current socio-cultural standards? Does carefully planned and created ambiance give a clear unambiguous sense of a place? Or, it is not ambiance but the processes of decoding and perception that result in various interpretations of the same experience of atmosphere?
\end{abstract}

\section{Introduction}

The aim of the study is to grasp and analyse the intangible process taking place between ambiance and perception. The paper discusses two concepts: ambiance and perception, in the context of the post-industrial heritage in Łódź, Poland. It is necessary to stress that the study focuses rather on the phenomena and relation between ambiance and perception as well as on senses than on tools. It draws research into a relationship between what is projected and actually experienced, between the tangible and the intangible and reveals the process of decoding ambiances where heritage plays a dominant role. In order to analyse the process, a brief introduction to background ideas should be presented.

\subsection{The concept of ambiance and perception}

The theory of ambiance is not new, and moreover, it has been developing dynamically over the past twenty years, focusing in particular on "material atmospheres, perceptive

\footnotetext{
*Corresponding author: anetta@p.lodz.pl
} 
configurations, physical phenomena, "affective tonality" of places and everyday practices of city dwellers. It lies at the very heart of any sensorial approach of inhabited spaces and reflects the sheer diversity of the senses (light, sound, heat, smell, air flows, kinaesthetic, touch, etc.)" [1]. As Bohme believes, the concept of ambiances occupies the space between subject and object [2]. Referring to Siret, since the past few decades, there has been a growing need "to grasp the architectural and urban aesthetic in all its human sensory dimensions, sonic, tactile, thermal, aeraulic, kinaesthetic or olfactive" [3]. This need is based on the belief that various sensory tonalities affect perception of built environments and their ambiances in which users of a given space are immersed.

It is worth noting here the difference between sensation and perception. Perception is understood as the effect of how the brain processes information from the senses. Hence, perception is multi-sensorial. Furthermore, the notion of ambiances makes us realise that our immediate perceptions of the world have both visible and non-visible components and therefore requires the consideration of all senses and their interplay in the process of perception.

As Thibaud states, "ambiances are both subjective and objective and thus involve people's lived experience as well as their physical environment" [4]. However, according to Pallasmaa, people feel atmospheres immediately and without being conscious of the process, since the ambiance of a given space is the overall feeling that tunes their minds in a specific way [5]. Another perspective has been presented by Morello and Piga [6]. The researchers claim the perception is based on the physical and sensorial experience of places, which includes a multiplicity of aspects, and contributes to the personal construction of the mental image of places. Their statement is based on Kevin Lynch's understanding of the image of a place as a mental construction derived from the analysis of a direct and indirect manenvironment relationship [7]. This logic allows to draw a conclusion that perception is a highly subjective notion.

\subsection{Theory of direct and indirect perception}

In psychology, there are two main theories of perception, direct and indirect, to be distinguished, and two types of processes in perception: bottom-up processing and top-down processing.

In an article titled "Visual Perception Theory", Saul McLeod wrote, "in order to receive information from the environment we are equipped with sense organs e.g. eye, ear, nose. Each sense organ is part of a sensory system which receives sensory inputs and transmits sensory information to the brain" [8]. Thus, the spontaneous activities of looking, listening, and touching, together with the satisfaction of noticing are considered to feature a direct perception [9]. According to Gibson, direct perception is not just a mode of cognition but "the simplest and best kind of knowing" [10]. In other words, Gibson's ultimate sense of direct perception is that of perception based upon information obtained through free exploration [11]. Gibson claims that perceiving is "an automatic response to a stimulus" [10].

On the contrary, some argue that perceptual processes are not direct, but depend on the perceiver's expectations and previous knowledge, as well as the information available in the stimulus itself. Psychologist Richard Gregory has proposed a theory of indirect perception [12]. According to his concept, stimulus information from our environment is frequently ambiguous, so to interpret it, we require higher cognitive information either from past experiences or stored knowledge in order to makes inferences about what we perceive. 
As it has been mentioned already, psychologists distinguish between two types of processes in perception: bottom-up processing and top-down processing. Gibson's theory reflects a 'bottom-up' idea while Gregory's vision presents a 'top-down' approach. The first concept is also known as data-driven processing, because perception begins with the stimulus itself. Whereas top-down processing refers to the use of contextual information in pattern recognition since it is assumed a context aids understanding [8].

\section{Case studies}

In all cases described in this paper, a natural ambiance was replaced by artificially made atmosphere to enhance the sensation of a place. Actually, it was the state of a building or of an urban space that induced an ambiance leading to perceptual experience and, in consequence provoked the action. Therefore, the creators of the ambiances built up a set of multi-sensorial experiences to allow immersion and the feeling of a place. The results contribute to the quest of architecture and environment envisioning and genius loci interpretation.

The research into ambiance and perception focuses on three aspects exemplified by carefully chosen case studies, viz.: a social aesthetic that examines shared sensorial experiences in the first case, on a physical context dealing with the propagation of signals in urban space in the second example, and on design that involves the sensorial aspects of built spaces in the last one.

\subsection{WIMA post-industrial interior awaking}

In 1877-1879, a large cotton factory was established by two industrialists, Juliusz Heinzel and Juliusz Kunitzer in Widzew - at that time a village at the outskirts of Łódź. Twenty years later the enterprise already consisted of a spinning mill, a weaving mill, a bleach mill, finishing and printing departments. There were own gasworks, a power station and a railway siding. The number of employees was over 3,500. Under the leadership of Oskar Kon - the new president and major shareholder - the company was transformed into a multi-branch combine in the $1920 \mathrm{~s}$. A decade later, the firm already employed 10,000 people and was considered as one of the most modern textile factories in Europe. After World War II, the nationalized enterprise was divided into three independent plants producing cotton goods, synthetic yarns, and textile machinery. The cotton production continued until 2009, when it was no longer competitive on the market, dominated by Asian yarn. This was the last cotton spinning mill in Poland [13]. 


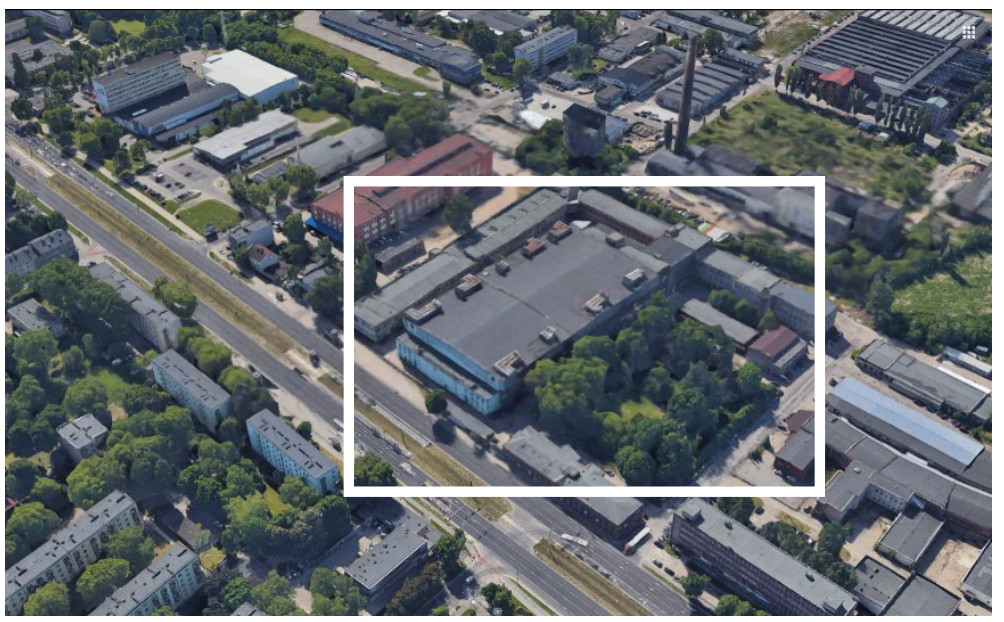

Fig. 1. The WIMA factory complex in Łódź. Source: Google Maps.

Since the collapse of production, a new use of the buildings has to be found. For the company owners the main asset and the burden at the same time is a huge three-story spinning mill. The building is one of the largest built in Łódź with an impressive width of 69 metres (fig. 1.). It is not surprising then that it is challenging to re-use it. The mammoth structure is surrounded by other post-industrial objects successfully let to a number of creative businesses - companies, foundations and associations dealing with culture and art. There are photographers, actors, filmmakers, designers, musicians, DIY enthusiasts and graduates of art schools in Lodz. As a result, recently the place has been re-branded officially from the Cotton Industry Company into the Creative Industry Company.

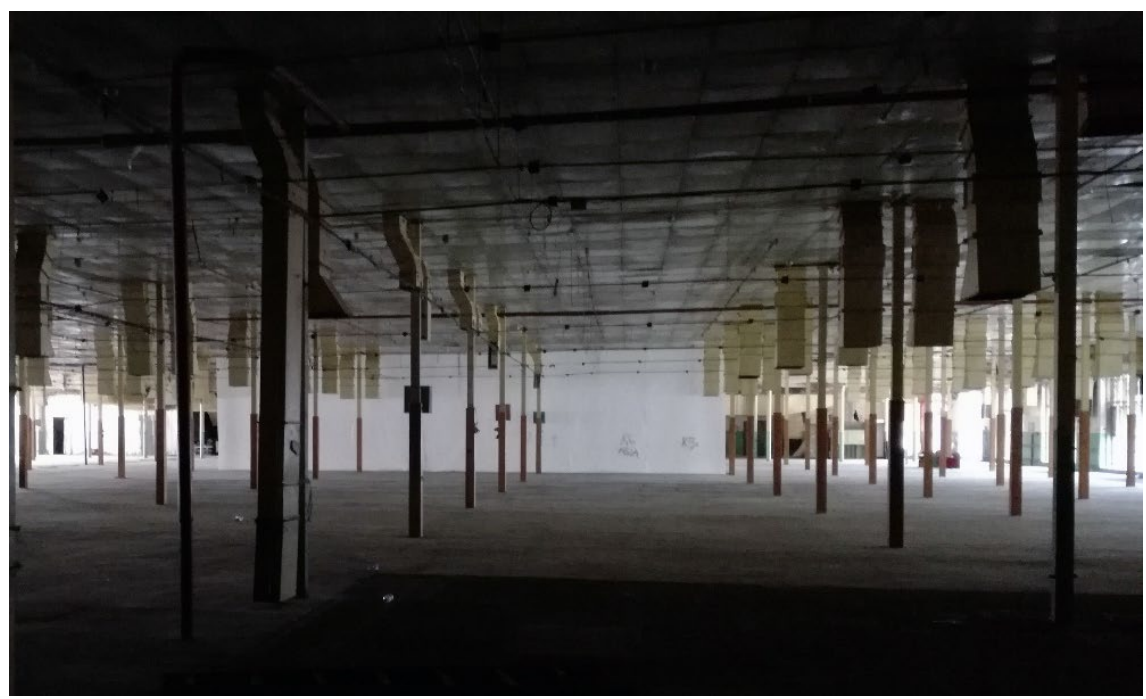




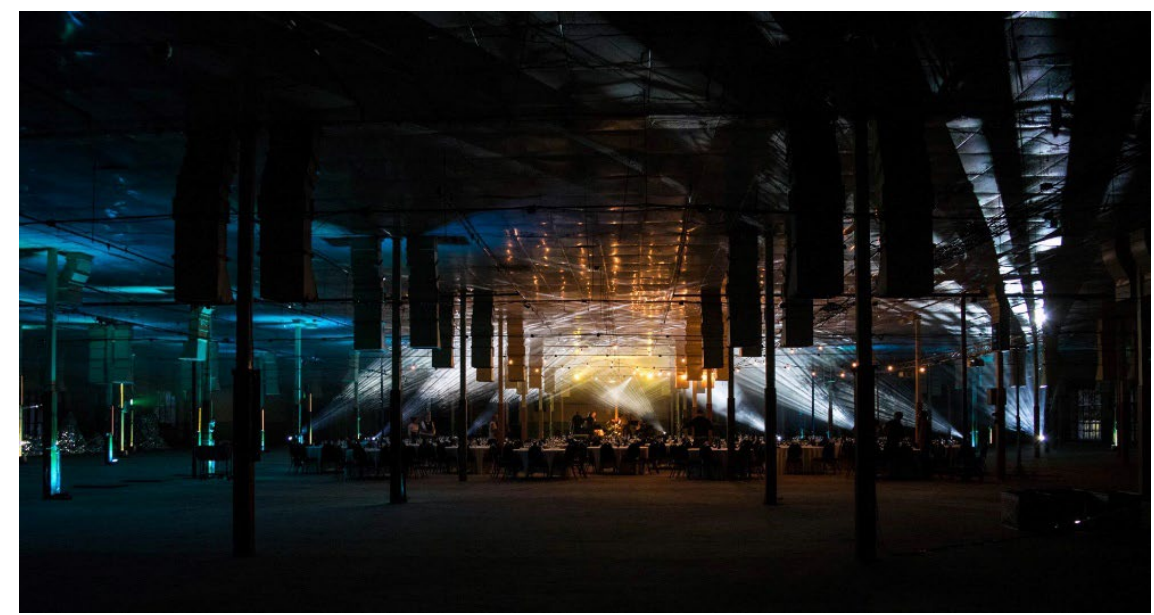

Fig. 2. The same interior of a disused mill in the WIMA complex - as it is usually (top) and during the described event (bottom). Source: eCAADe 2018 archive.

For the purpose of this study, a perception of an abandoned textile mill interior has been confronted with the immersive experience of the same space during a social event (fig. 2.). The empty unused interior by the means of light and sound effects as well as real objects was transformed into an eclectic environment, balancing between past, present and future (fig. 3.). A multifaceted appearance was matching the nature of the local - strongly eclectic heritage, reflecting diverse cultural roots of the city dwellers. Ultra-modern magnetic light effects contrasted with historical costumes of dancers who were giving lessons of Polonaise (Polonez), the national Polish traditional dance, to the audience; a delicious smell of food representing four cultures that constituted the heritage of Łódź, viz.: Polish, German, Jewish and Russian; a jazz music band evoking sentiments to the prosperous times of the past, yet to the birth of modern times - all together and interfering with each other built the richness of multisensorial atmosphere and generated unique moments of shared social sensorial experience (fig. 4.).

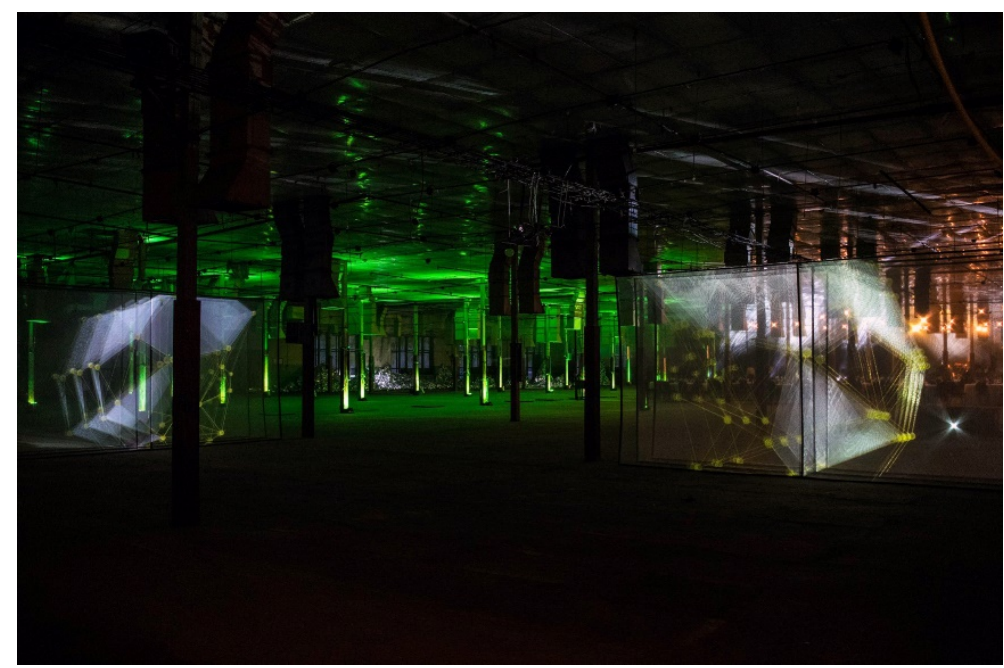

Fig. 3. The interior transformed by the means of light and sound effects as well as real objects. Source: eCAADe 2018 archive. 


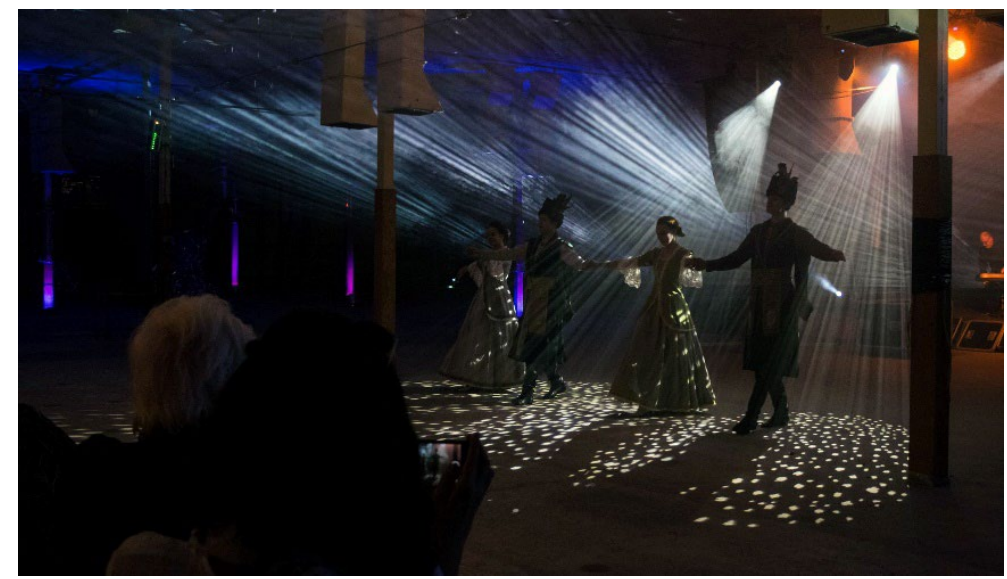

Fig. 4. The lesson of of Polonaise (Polonez), the national Polish traditional dance in the ultra-modern magnetic lights. Source: eCAADe 2018 archive.

There is no doubt that, in this case, perception was based on the culture and heritage literacy. By decoding the meanings, the ambiance offered a number of layers unveiling to individuals, depending on their background and comprehension abilities without affecting overall attractiveness. As the event gathered over 200 participants from more than 40 countries from all over the world, for some foreigners it could be just exotic or intriguing experience, while among local residents or those representing the four mentioned cultures, it could stimulate a feeling of deeper reflection or even a patriotic elation. However, what would be their (visitors and locals alike) perception of the same place, if they found themselves in a room full of operating textile machines, producing unbearable noise, dust and smell? Or what would be their perception of this production room as it is nowadays with countless layers of paint peeling off the wall, piles of unidentifiable rubbish in the corners and a dead pigeon on the floor? The set of reflections brings yet another questions: are we into the heritage as it was, or as it is at present, or rather to its projection, tuned to current socio-cultural standards? Does carefully planned and created ambiance give a clear unambiguous sense of a place? Or, it is not ambiance but the processes of decoding and perception that result in various interpretations of the same experience of atmosphere?

\subsection{Light Move Festival}

Another issue to be discussed is the ambiance of special open events and its role in promoting local heritage. This case refers to a physical context dealing with the propagation of signals in urban space. It will be illustrated with the immense success of a locally organised Light Move Festival, the most important event of this kind in Poland. It is worth mentioning the idea of organising such festival in Łódź has been strongly inspired by the example of la Fête des Lumières in Lyon [14], Łódź partner city. What is interesting is that kinetic art and 3D mapping on historical facades, offering local heritage-related storytelling, are among the most popular installations during the festival [15]. They are appealing for a broader audience, and each year there are hundreds of thousands of visitors to the city, who for three days admire unique visual effects on facades of historic buildings. According to the local press, this event has already contributed significantly to the change in perception of Łódź from the gloomy post-industrial city into an attractive, and (literally) colourful place. At the same time, and by using the same means the same event encapsulates different message to members of a local community. It offers a specially prepared ambiance, touching heritage-related sentiments, and strengthening the feeling of belonging to the place, of identity, safety and 
continuity. Raising awareness through events of this kind may contribute significantly to a process of urban regeneration and indicate values of architectural environment, which remain unnoticed or even disobeyed by a local society during their everyday walk of life.

\subsection{Revitalisation project of Lürkens factory in Łódź}

The third case study presents a master diploma design, successfully submitted and defended by architecture student Katarzyna Romanowicz, entitled: "Lürkens' Spinning Mill - A project of converting the former knitwear factory of Wilhelm Lürkens into a creative coworking space", supervised by the author of this paper.

Wilhelm Lürkens' residential and industrial complex is located in Łódź, in the southern part of a small urban block limited by the following streets: from the north -6 Sierpnia Street, from the south - Andrzej Struga Street, from the west - Wólczańska Street, and from the east - Kościuszki Avenue. The premises are located in the inner city centre, in the immediate vicinity of Piotrkowska Street, the main historical axis of Łódź (fig. 5.).

The Lürkens factory belonged to medium-sized textile enterprises in Łódź. The employment did not exceed 150 people even when cotton production was booming. The industrial, commercial and residential buildings were built in three stages between 1889 and 1912. From the very beginning, the production activities took place along Wólczańska Street, while the residential and administrative function was concentrated along Aleja Spacerowa (now Kościuszki Avenue). In terms of architecture, the most interesting is the complex of a spinning mill with a water tower, adjoining boiler and engine house, and a high factory chimney, erected at the end of the first decade of the $20^{\text {th }}$ century. These objects have received a more careful architectural treatment as compared to the more severe objects from the earlier period. Facades have the features of late Art Nouveau, with simplified decorative details in the spirit of early modernism.

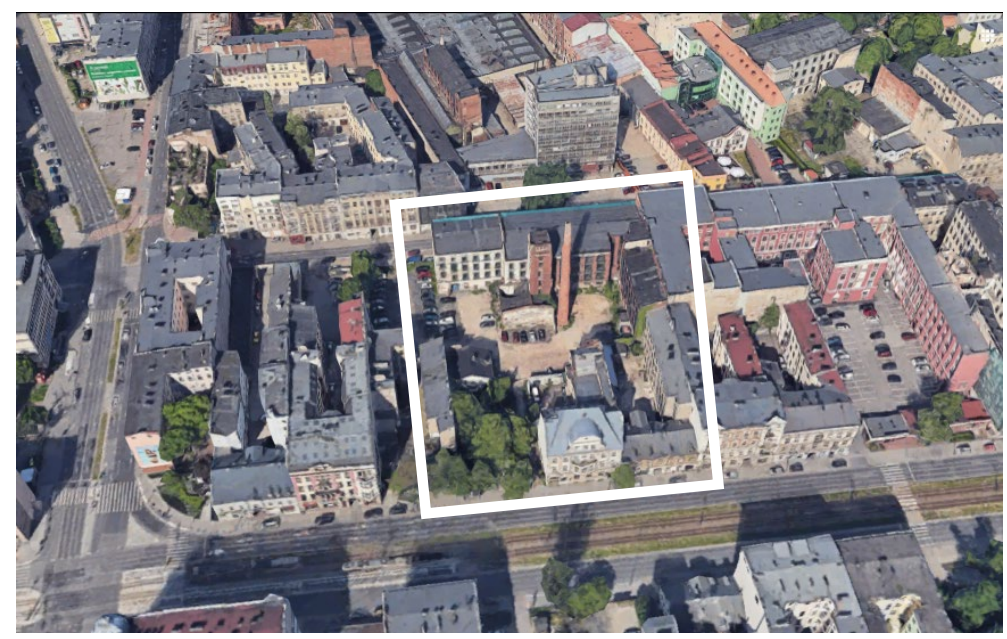

Fig. 5. The Lürkens factory complex in Łódź. Source: Google Maps.

After 1945, the factory was nationalized. Since 1969, Polboy clothing factory operated there, later incorporated into Próchnik, a company specializing in men's wear, mainly produced for export. In 2006, industrial buildings were bought by Spanish investors with an aim to convert them into a hotel. These plans have not been implemented until today, and buildings are deteriorating [16]. 

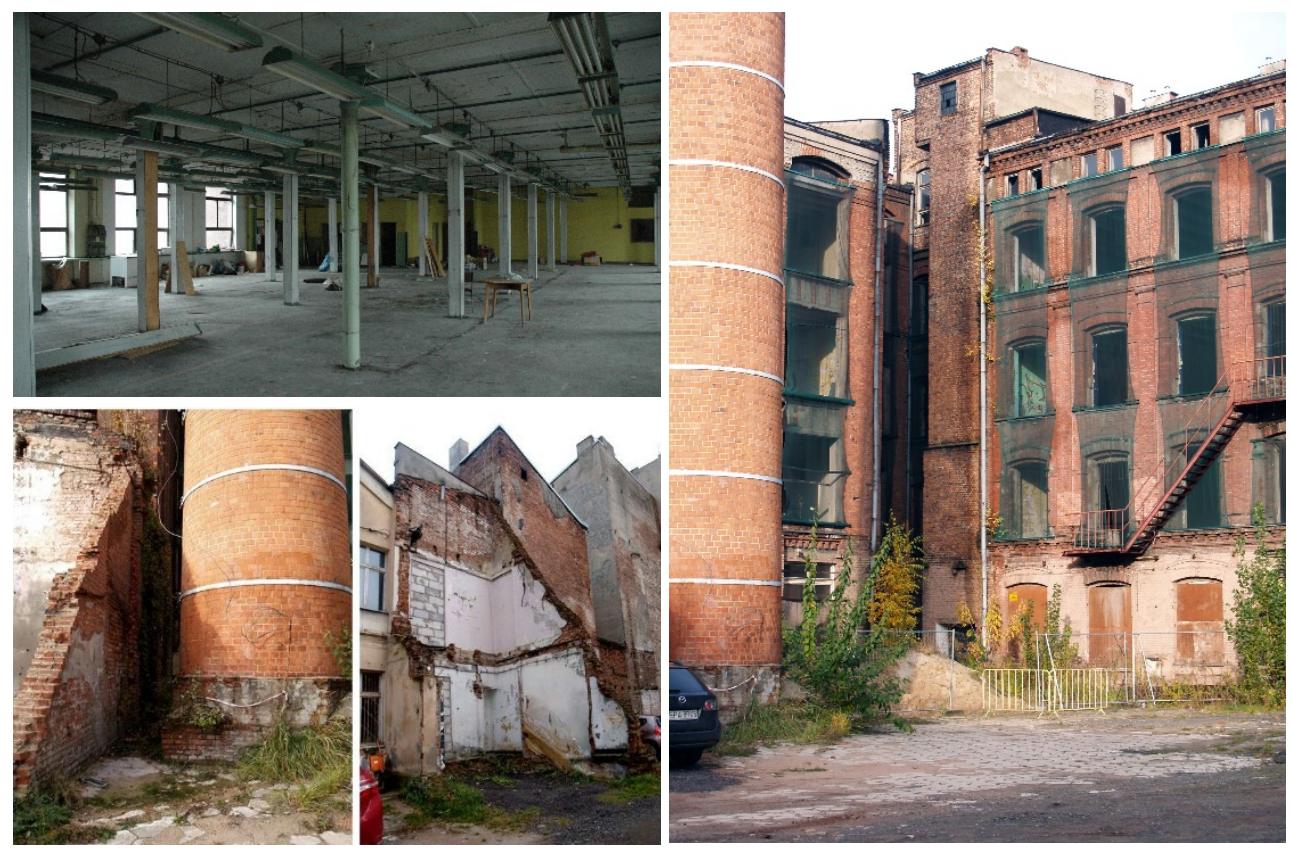

Fig. 6. Dilapidated buildings of the Lürkens factory complex in Łódź. Source: images by Katarzyna Romanowicz and Bartosz M. Walczak.

The state of the buildings has been getting worse with each year and some parts of the factory complex have already collapsed. Uncontrolled wild greenery started to grow interfering with existing structures (fig. 6.). Therefore, the project of converting the Lürkens' built legacy into a dynamic, modern and full of life place required deep studies, understanding of a history and above all, grasping the image of a place to create an ambiance that would attract visitors and potential business.

A palette of artistic means was used to (re-)create the ambiance of abandoned factory waiting for successful revitalisation. So, the study covered aspects of searching for reminiscences of the past, the atmosphere of the place, both in urban courtyards and architectural interiors, and original materials that would influence the perception of the physicality of the place. However, the idea was not to reconstruct the original genius loci of the place but to define a set of attractors addressed to contemporary society and taste. Therefore, a proposed design emanates with neat and cosy colours and lights. Furthermore, the refreshed post-industrial space was furnished in a non-invasive modern style what might evoke a feeling of comfort and high quality (fig. 7-8). 

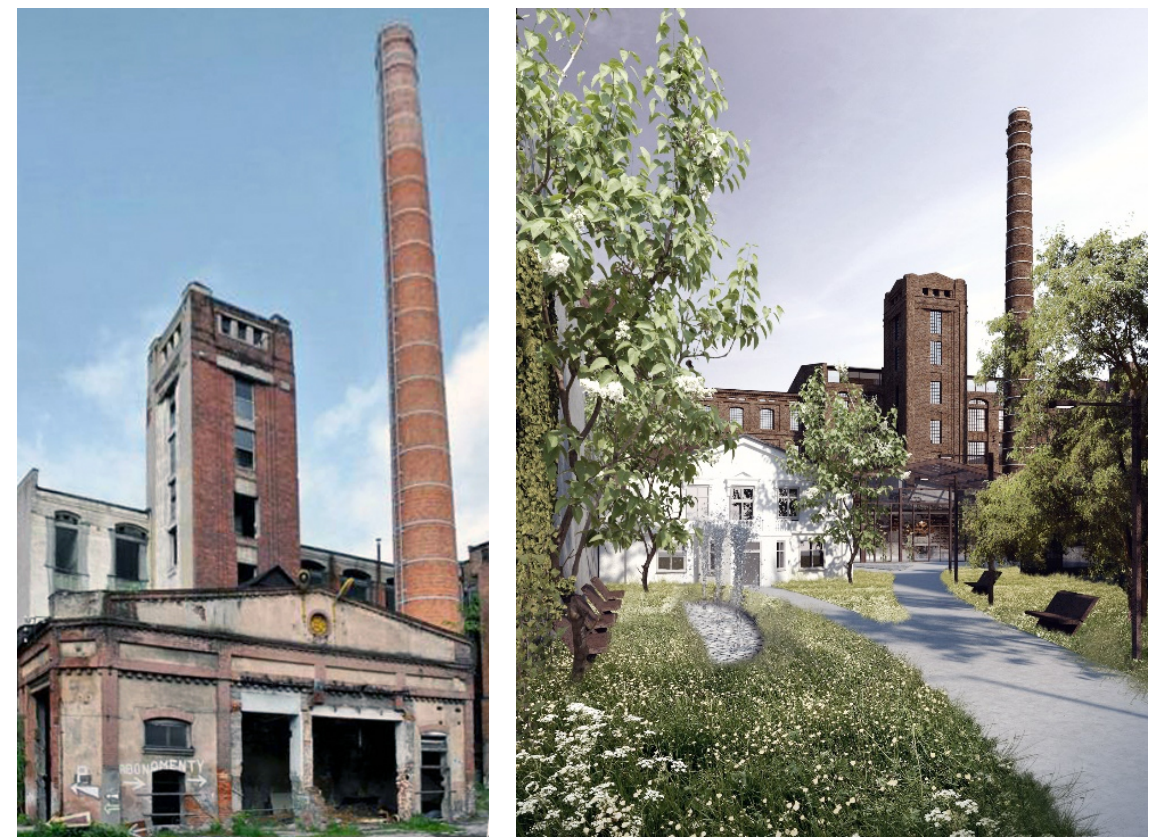

Fig. 7. Main building - current state (left) and envisioned future (right). Source: images by Katarzyna Romanowicz.

Recalling the past literally is not what is expected. To expand on it, there is a textile machine in the Central Museum of Textiles in Łódź operating during special shows. The noise a single machine makes is unbearable. Behind the machine, on the wall, there is a visual projection of the mill interior filled with working machines. The scenery informs clearly about the noisy working environment. Moreover, the smell of oil and metal reminds of another negative characteristic that would not be appreciated nowadays. Therefore, the careful selection of sensorial factors in the project led to a decision to enhance urban and architectural space perceived visually, whereas omitting less attractive elements of the past realm. To sum up, the project positioned the past legacy as an anchor and a link to local identity but let imagination to supplement the dominant visual stimulus.

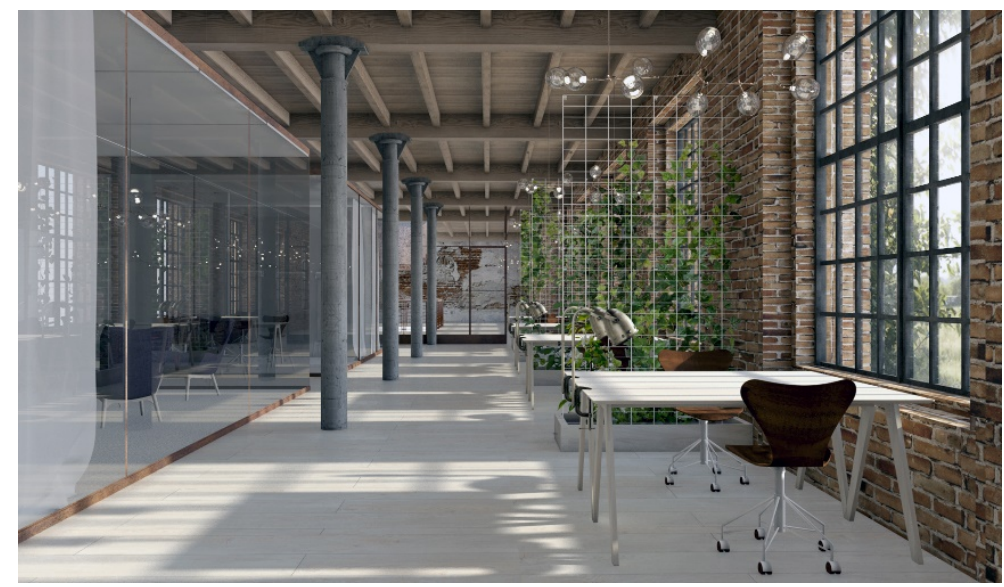




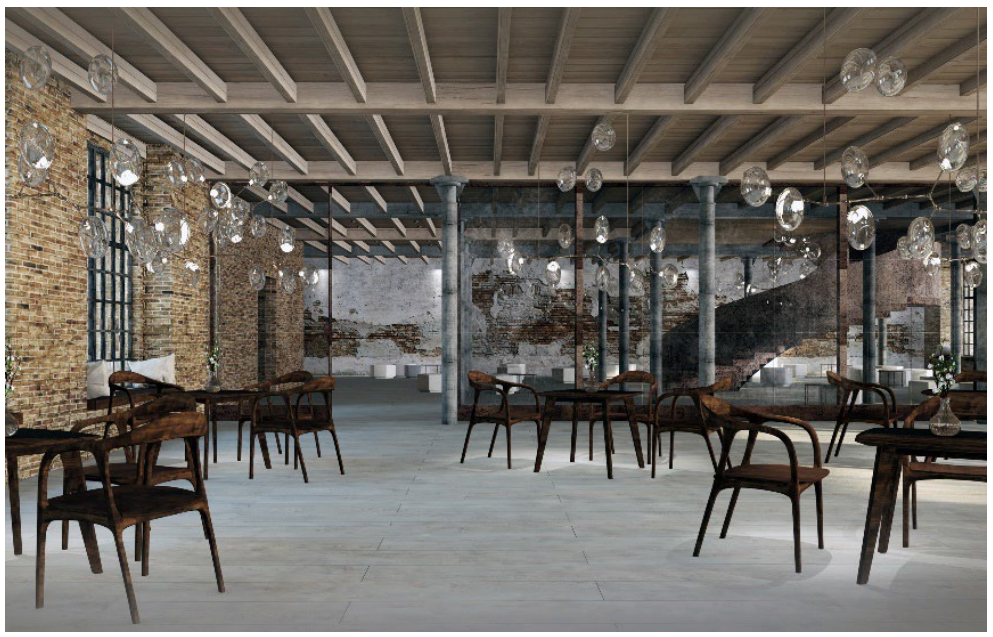

Fig. 8. Modernity inspired by post-industrial decay - vision of re-used interiors of the Lürkens factory. Source: images by Katarzyna Romanowicz.

\section{Discussion and concluding remarks}

Selected case studies allowed to analyse aspects of sensorial stimulation, such as light, sound, or smell and to investigate the embodied and affective experience of spatial settings.

The relationship between the place, its atmosphere and its perception by various audiences has been observed. It allows to drive the first conclusion that the ambiance acts as a source for various modes of perceiving a given place. However, what ambiance encapsulates is not equal to perception. In this regard, ambiance should be understood as a state or aura as opposed to perception assumed as a process.

The transition of sensorial immersion from ambiance to perception requires the process of decoding. Decoding might be explained as the process running between ambiance and perception and, furthermore, leading to understanding. Therefore, the outcome of the overall process is of cognitive characteristics. However, either we consider direct or indirect perception, to run such process a decoding key is necessary. The case studies, described in previous paragraphs, underline the meaning of the process of decoding ambiances where heritage plays a dominant role.

The ambiance of a place can be natural or enhanced with some additional sensorial effects, or, even more, it can be artificially created. The purpose of the latest one can be to evoke sensation, mood, emotions, reactions, memory and alike.

Considering the ambiance created intentionally, a palette of stimuli it offers might not be the same as what is absorbed. In other words, a creator of the atmosphere might not succeed with communicating intensions. Furthermore, ambiance can be perceived totally or partially or not noticed at all since the perception skills depend on personal abilities, experience and knowledge.

Decoding can be a background action, uncontrolled and free, often happening on emotional level. Even though, the resultant processes of interpretation are knowledge and 
cognitive-based. They rely mainly on abilities to associate direct and indirect past experiences.

Even if ambiance is composed of a selective sensorial palette, as described in the third case study, the post-industrial legacy is recognised as the heritage to be protected and as the anchor of a local identity. The anchoring in the past gives a sense of security, continuity, identity. It is associated with the issues of sentiment, looking at the past as if it was better than present times and therefore choosing "flavours" and genius loci pre-set ambiance to build an encoded image of a place.

To conclude with final remarks, the representation of atmospheres is a powerful tool to understand the immaterial aspects of lived space. In the respect of cultural heritage, through creation of ambiance, such rich envisioning experience can integrate knowledge about the place, time, society, factors affecting space, as well as other elements that shape the cultural environment.

\section{References}

1. https://www.ambiances.net/international-ambiances-journal.html

2. G. Böhme, Atmosphere as the fundamental concept of a new aesthetics, Thesis Eleven 36, 113-126 (1993)

3. D. Siret, Understanding past sensibility to grasp present architecture: the example of solar radiation in: A. Kepczynska-Walczak (ed.), Envisioning Architecture. Image, Perception and Communication of Heritage (2015)

4. J-P. Thibaud, The sensory fabric of urban ambiances, Senses \& Society, 6 (2), 203-215 (2011)

5. M. Amundsen, Q\&A with Juhani Pallasmaa on Architecture, Aesthetics of Atmospheres and the Passage of Time, Ambiances (2018)

6. E. Morello, B.E.A. Piga, The Image of Productive Landscapes: A Method for the Perceptual Analysis of Industrial Heritage in: A. Kepczynska-Walczak (ed.), Envisioning Architecture: Image, Perception and Communication of Heritage (2015)

7. K. Lynch, The Image of the City (1960)

8. S. McLeod, Visual Perception Theory (2008)

9. J.J. Gibson, The senses considered as perceptual systems (1966)

10. J.J. Gibson, The ecological approach to visual perception (1979)

11. A. Costall, A. Still, Gibson's Theory of Direct Perception and the Problem of Cultural Relativism. Journal for the Theory of Social Behaviour, 19:4, 433-441 (1987)

12. R. Gregory, The Intelligent Eye (1970)

13. B.M. Walczak, Zespoły fabryczno-mieszkalne w europejskim przemyśle włókienniczym w latach 1771-1914 (2010)

14. http://www.fetedeslumieres.lyon.fr/en (access: 20.10.2018)

15. http://www.lmf.com.pl/\#/page/14 (access: 20.10.2018)

16. J. Kusinski, R. Bonislawski, M. Janik, B.M. Walczak, Łódź. Księga fabryk (2016) 